\title{
Non-destructive, Multi-scale 3D Fractographic Analysis of a Carbon Fiber Composite Hockey Stick after Compressive Failure
} Jeff Gelb, Will Harris, Naomi Kotwal, Wayne Broderick, Leah Lavery, Hrishi Bale, and Arno Merkle

${ }^{1 .}$ Carl Zeiss X-Ray Microscopy, Inc., Pleasanton, CA, USA

Composite materials are present in many areas of daily life. From bicycle frames and automotive components to aerospace and industrial applications, composites are widely employed to produce tough materials that make the best use of the constituent material properties. However, fractures and failures occur in a variety of modes stemming from extreme and varied loads that are a natural consequence of the material service conditions (such as tension, compression, or bending modes). Understanding the fracture mechanics is critical to future improvements of the material design.

Failures in fiber-reinforced composite materials are often the result of delaminations occurring at the interface of fiber-rich and resin-rich regions. While these failures are known to occur and are often encountered in computational simulation studies during composite design, visualizing and characterizing the nature of these cracks is extremely challenging using conventional techniques. Traditional characterization with light- and electron-microscopy require 2D polished sections in order to produce high quality images of the failure sites, which require some knowledge about the failure location prior to performing the characterization. The failures are also often complex 3D cracks that wind around the fibers, which require a 3D characterization protocol in order to be properly understood.

In the present study, a section from a commercial carbon-fiber composite hockey stick was subjected to compressive load, in order to induce cracks within the structure. A multi-scale 3D investigation was then performed using the technique of X-ray microscopy (XRM), employing the computed tomography (Micro-CT) approach in tandem with higher-resolution X-ray imaging. The micro-CT approach was employed to gain an initial depiction of the entire device, showing the various fibers in 3D and locating a region that suggested cracking/failure. A higher resolution objective was then used to nondestructively "zoom in" on this region and produce a 3D XRM volume of this targeted region of interest, which revealed the precise nature of the cracking with single-micron resolution. The crack openings vary from the single micron up to the tens of micron scale, and at isolated and distinct regions along the curved portions of the wall of the hollow structure. It was observed that the primary cracking occurred along the fiber domain boundaries as a delamination effect, with some smaller cracking bisecting the domains in a crack bridging effect. The primary cracks follow the contour of the wall, again supporting observation of the delamination between plies of approximately 100 micron thickness. Through visualization and analysis, the size and shape of these cracks was characterized in $3 \mathrm{D}$, which may be used for further modeling and simulation studies in designing newer, more robust composite materials for this particular application.

References:

[1] A P Merkle and J Gelb, Microscopy Today (2013) pp. 10-15.

[2] M Feser et. al., Measurement Science \& Technology 19 (2008) p. 1-8. 


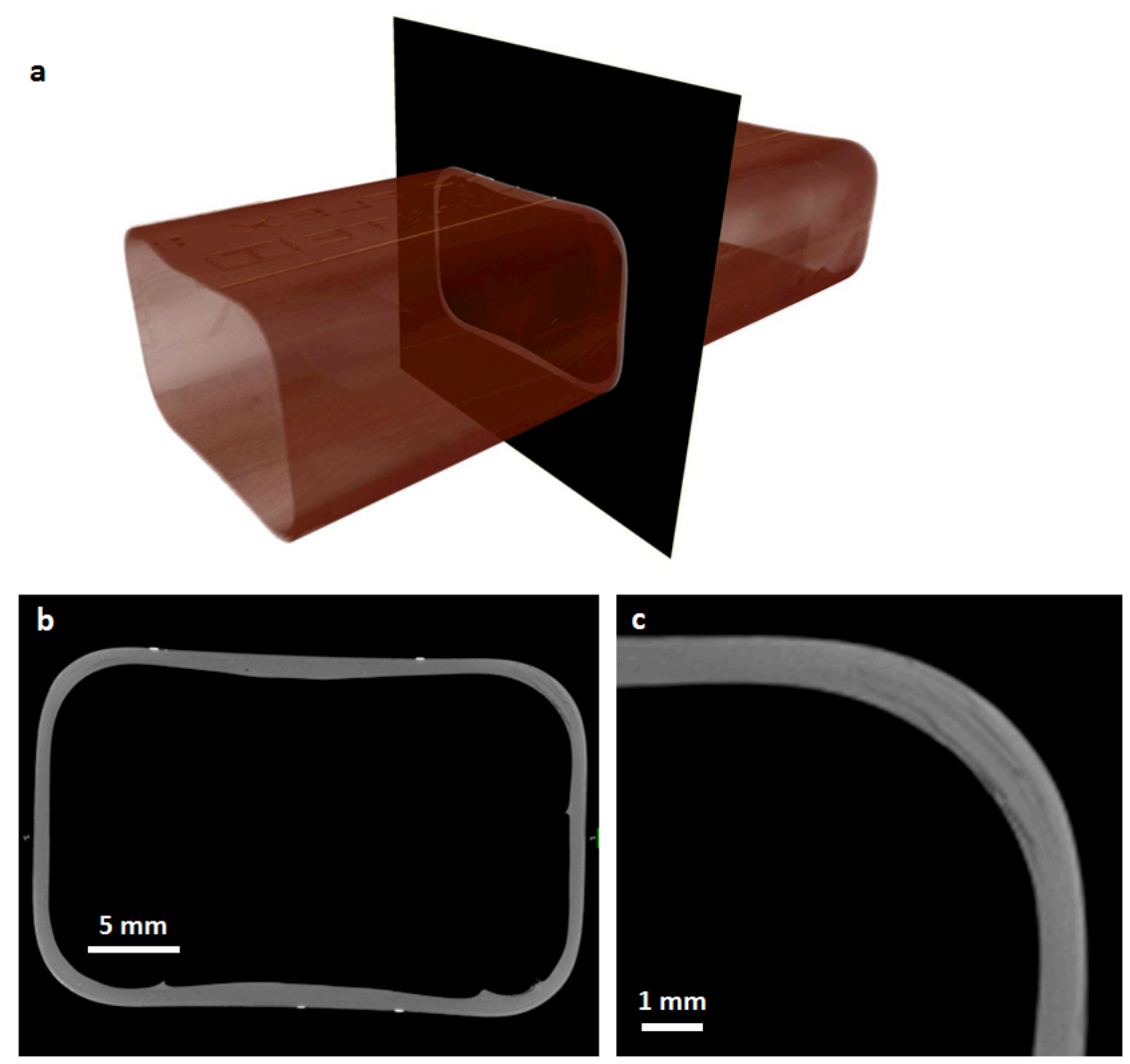

Figure 1. Initial 3D X-ray imaging of the hockey shows indications of defects nucleating in highly localized regions, and not throughout the entire material.

\section{Zoom with Multiple Objectives}

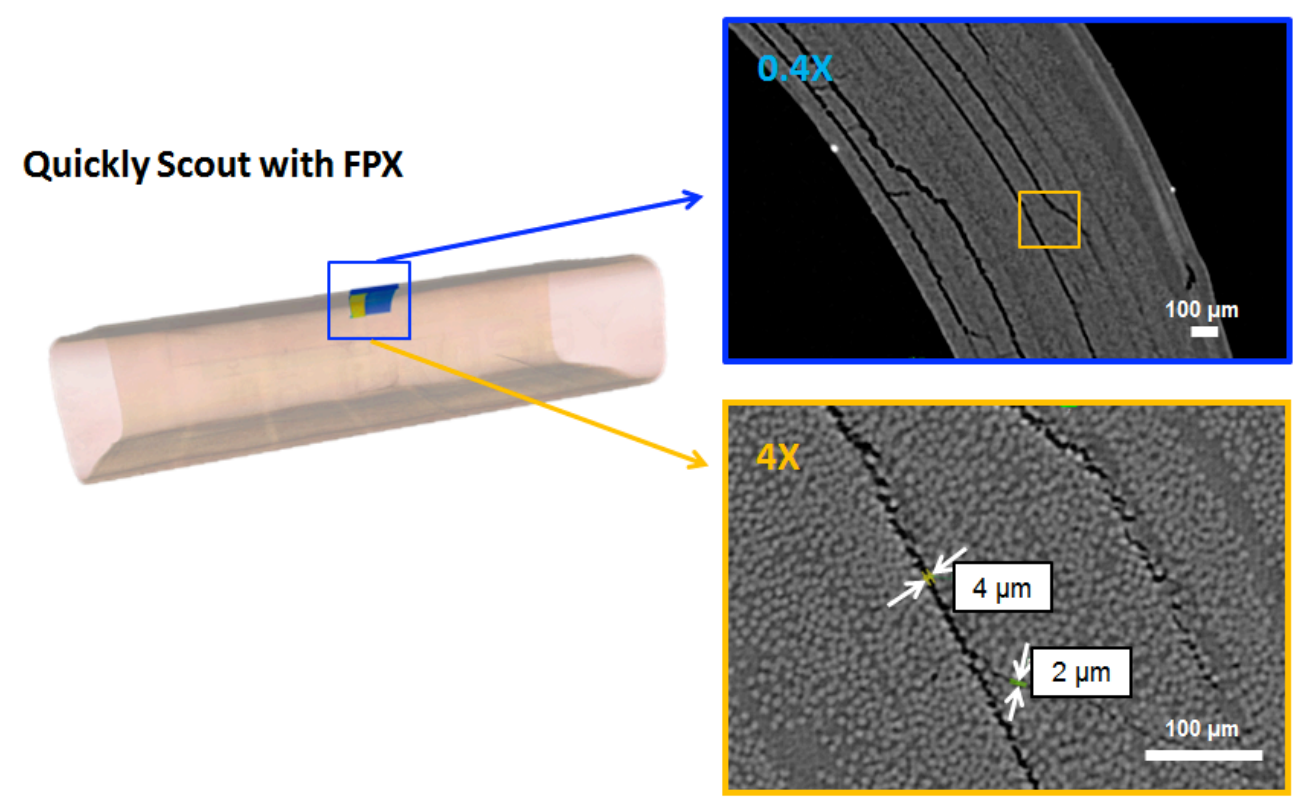

Figure 2. Zooming-in with high-resolution 3D X-ray microscopy reveals the precise nature of the defects, between fibers and along fiber domain interfaces. 